\title{
Meeting Report: ASM Candida and Candidiasis
}

\author{
Joy Sturtevant \\ Louisiana State University School of Medicine, Department of Microbiology, Immunology, and Parasitology, \\ Center of Excellence in Oral and Craniofacial Biology, 1100 Florida Ave, Box F8-130 New Orleans, Louisiana \\ 70119
}

Received 20 May 2004; accepted in revised form 20 May 2004

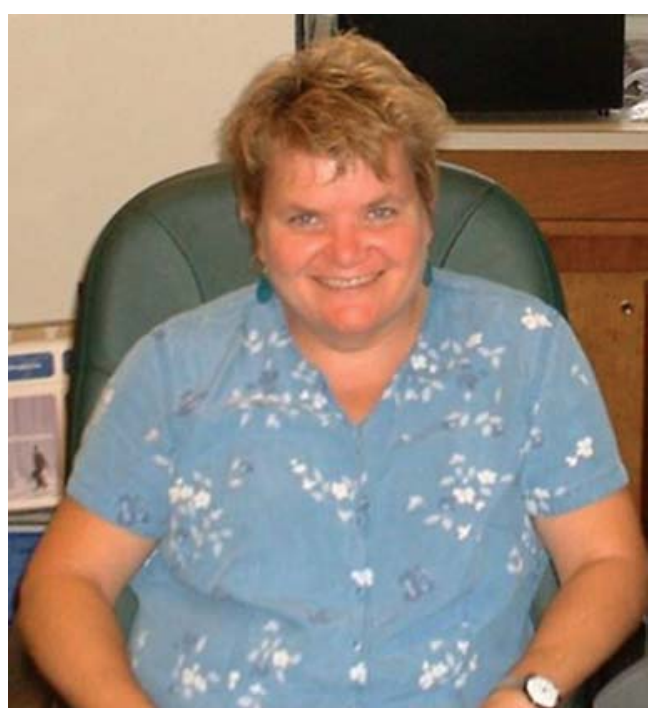

The 7th Americal Society of Microbiology (ASM) Candida and Candidiasis Conference was a well organized meeting held in Austin, Texas in March 2004. The meeting began with comprehensive overviews of what we know about Candida in the areas of basic, clinical, and pharmaceutical research presented by Drs. Soll, Rex, and Hitchcock [1]. Throughout the 4 days, all aspects of present Candida research were covered. In order to emphasize current studies, only two speakers in each session were predetermined; the remaining three were selected from submitted abstracts. Three clinical presentations challenged basic researchers to redirect their experimental approaches to address existing problems in the wards. There was an outside the box session led by Drs. Casadevall and Haynes on "Candida and its hosts", which took the virulence session from the Conference in 2002 one step further. They chal- lenged our concepts of how we classify ourselves as scientists ('gene bashers' or 'host defenders'); and on why/when we use animals in experimentation. There were workshops from NICDR on funding, new developments by Merck, and a progress report on the Candida annotation working group. Just in case we weren't learning enough about Candida there were three poster sessions, which included close to 250 presentations. Finally, there was plenty of opportunity to discuss Candida and all its aspects at lunch and down at the bar. Before I begin an overview of the scientific content of the meeting, I start with a disclaimer. It will be impossible to discuss, let alone mention every significant observation reported in this meeting. However, I will try to touch on the main topics that were discussed in the context of both Candida and candidiasis.

Perhaps the greatest boost to our current research is the development of new tools to address old problems. However, we must remember to ask questions before deciding on corresponding experimental procedures. These questions should not only address biological, but also clinical significance. No model will be perfect to answer all the mysteries of candidiasis but specific models will supply important information if the appropriate question(s) is asked. The clinical overviews presented by Drs. Ruhnke, Sobel, and Edwards, confirmed that more work needs to be done, and epidemiological studies also tell us there is still a problem. Those that research with C. glabrata and C. parapsilosis are certainly still in business. However, we are not much better off with treatment strategies and certainly no better off with diagnosis.

At first glance of the program, one would think that there is not much work on the level of the host 
response. Current research seems to focus on Candida rather than candidiasis. In actuality, the molecular biologists are beginning to explore host- fungi interactions and these will be discussed below. Historically CD4 ${ }^{+} \mathrm{T}$ cells and neutrophils have been associated with oral and disseminated candidiasis, respectively. However, CD4 ${ }^{+} \mathrm{T}$ cells are not the complete answer to host immunity to oral candidiasis. Resistance/ susceptibility to vaginal candidiasis has not been associated with an immune defect. However, studies now indicate that a hyperactive immune response as opposed to no response seems to correlate with vaginal candidiasis. New components are being identified that play a role in host immunity including Langerhans cells, Toll like receptors, dectins, defensins, as well as epithelial cells themselves having fungistatic activity (P. Fidel, D. Lewandowski, J. Naglick, M. Netea, E. Adams, D. A. Schofield). Even though the studies of the host immune response to Candida is "negligible" compared to fungal response studies, there is compelling, and perhaps not surprising evidence, that the genetics of the host is what makes the true difference (P. Fidel, D. MacCallum) (1).

Prefacing much $C$. albicans work is that Candida is a difficult organism to work with genetically. There is now a greater selection of available knockout strains and systems that may allow large scale screens. We now have a selection of nonnutritional markers including NAT and hygromycin B. Multiple GFP reporter systems have been developed for functional, localization, and expression studies. Whereas before, in vivo studies were mostly limited to survival and colonization, it is now possible to study the expression and action of single genes using GFP and the TET regulatable promoter system. (S. Noble, J. Wendland, C. Russell, J. Kohler, O. Reuß, B. Wong, C. Barelle, C.Gale, Y. Mao, S. Kauffman, S. Saville). However, the greatest tool which has been given to the Candida community is the sequencing of the Candida genome [2]. This was a large accomplishment initiated by Dr. Scherer and then continued and completed by the Stanford Genome Technology Center (http://www-sequence.stanford.edu/group/Candida/). At the 2002 Candida conference, an Annotation Working Group was inaugurated and given the onerous task of organizing all the current annotation groups in order create one uniform Candida genome data- base. In 2 years, they have done it! (temporary base: http://Candida.bri.nrc.ca/Candida/index. cfm? page $=$ CaAnno) (A. Nantel). Thanks must be given to the Pharmacia Corporation, the Burroughs Wellcome Fund, the Wellcome Trust, and the NRC Genome-Health Initiative as well as the Candida community for support of these accomplishments. In July 2004, the handling of the annotation website will be passed onto the newly formed Candida Genome Database (CGD) (http:// www.Candidagenome.org) (M. Costanzo). The CGD have accepted the task of keeping the annotation "literally" up to date. The availability of additional databases that have been developed for the Candida community were also presented (http://genolist.pasteur.fr/CandidaDB/) (C. D'enfert) and (http:/candida.bri.nrc.ca) (A. Nantel). Now that the $C$. albicans genome has been sequenced, what can we learn from other genomes? Initially, one would think that sequencing $C$. dulbiniensis would not be necessary since the two species are over $90 \%$ similar. However, C. albicans is significantly more effective at causing disease. $C$. parapsilosis is especially virulent in neonates and forms biofilms under high glucose conditions. Therefore, sequencing these genomes may accelerate the identification of genes involved in pathogenicity (D. Sullivan, G. Butler). Genome sequencing of five other Candida species is also being initated at the Fungal Genome Institute at MIT (C. Cuomo). Now our challenge will be to utilize these tools to hasten the pace of development of new antifungal therapeutic treatments and diagnostics.

The sequencing of the Candida genome identified the presence of mating type loci and, by genetic manipulation, Candida strains can now be induced to mate [3-5]. It is also clear that switching is required for mating $[6,7]$. The regulation of these events are being tackled and will certainly be the subject of future meetings (S. Lockhart, D. Davis). Dr. B. Magee reported that C. albicans can even mate in vitro with $C$. dubliniensis to form "C. dublicans". Does the ability of C. albicans and C. dubliniensis to mate cast doubt as to whether they are separate species? Cell biology studies have shown that $C$. albicans form mating structures similar to Saccharomyces cerevisiae, yet $C$. albicans does not appear to undergo meiosis [8]. Genes homologous to $S$. cerevisiae meiosis genes have been identified; some of these can complement 
2004 Foci

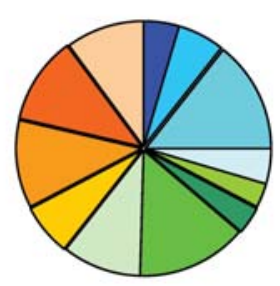

\begin{tabular}{ll}
\hline Mating & Epidemiology and Populations \\
$\square$ Genomics and Proteomic & Miscellaneous \\
a Clinical and diagnostics & a Host response \\
a Pathogenesis & Drug resistance \\
Biofilms & Morphogenesis \\
$\square$ Signaling & $\square$ Cell wall \\
\hline
\end{tabular}

Figure 1. Chart depicts grouping of abstracts for 2004 conference.

Saccharomyces, therefore what is missing (J. Bain, A. Bito)? Since it doesn't evolve by meiosis, it must use other mechanisms for adaptation. Differential gene expression is one solution. A vast number of transcriptional profiling studies are being performed to address Candida's response to hostile environments. Silencing and chromatin rearrangement are also possibilities (A. De las Peas, A. Dominguez). However, these mechanisms are also used by organisms that reproduce sexually, how else may Candida evolve? Several laboratories are now focusing on the genomic changes in $C$. albicans including loss of heterozygosity and genomic instability (G. Larriba, A. Forche, A. Selmechi, A. Davidson). Dr. M. Santos has elegantly addressed the evolution of the "mistranslation" of the CUG codon in C. albicans by expressing the Candida tRNA in $S$. cerevisiae. The study indicated that ambiguous decoding results in significant changes in gene expression and genomic instability. The decoding of CUG to serine may have played a significant role in the evolution of $C$. albicans. Does Candida willfully "mistranslate" CUG in order to adapt? Do these genomic changes help Candida or the host? Evidence was presented that loss of heterozygosity is often associated with increased potential to mate, acquisition of drug resistance, and increased adherence. This would indicate that genomic instability aids Candida. However, these phenotypes are easily assessed. Would we even see changes that favor the host since these strains would be less viable? Dr. Anderson addressed the question of haploid vs. diploid survival and corresponding abilities to respond to change (mutate). There are obvious pros and cons to each situation but since haploid species, such as $C$. glabrata, have a putative adaptation advantage at high drug concentrations, should this be taken into consideration in the wards?

The increase in fungal infections has often been attributed to the acquisition of drug resistance. Certainly there has been a demand for new antifungal drugs other than azoles. Caspofungin is the only new drug that has been approved recently and time will tell whether resistance to the echinocandins will evolve. Consequently, Dr. Edlind's group, in the spirit of learning from history, is purposely making caspofungin - resistant strains in order to identify mechanisms of resistance before it occurs in the clinic. At the previous meetings, presentations focused on the mechanisms of azole drug resistance: differential expression of drug resistance genes and/or mutations of genes in ergosterol biosynthetic pathways. This year, the focus was on what actually triggers or regulates the activation of the CDR and MDR genes. Elegant systems have been designed to study transactivation of the CDR and MDR genes and these tools have been used to study co-regulation and expression in clinical strains (M. Raymond, C-G. Chen, P. Riggle, A. Coste). However, is drug resistance truly a problem in candidiasis in the wards? Studies indicate that the increased incidence of infections due to non-albicans Candida species is may be due to drug resistance but that this is not a significant problem with $C$. albicans (M. Pfaller, J. Vasquez). How is this being determined? If the drug resistance genes are sophisticatedly regulated as these researchers hypothesize, will we be able to mimic activation states in vitro, in the clinical testing laboratory? Will these studies help us in the clinic? Can we use this information to design analogs, or perhaps synergistic treatment regimes so that resistance will not be initiated?

For that matter, are there other cellular processes in Candida that can be exploited as an avenue to design combinatorial therapy? What about intracellular vesicular and vacuolar pathways and protein transport? These processes are understudied in fungi, but the new sophisticated tools particularly the GFP reporter systems should greatly aid in dissecting these pathways (Y. Mao, P. Sundstrom, R. Eck, G. Palmer). Biofilms are 

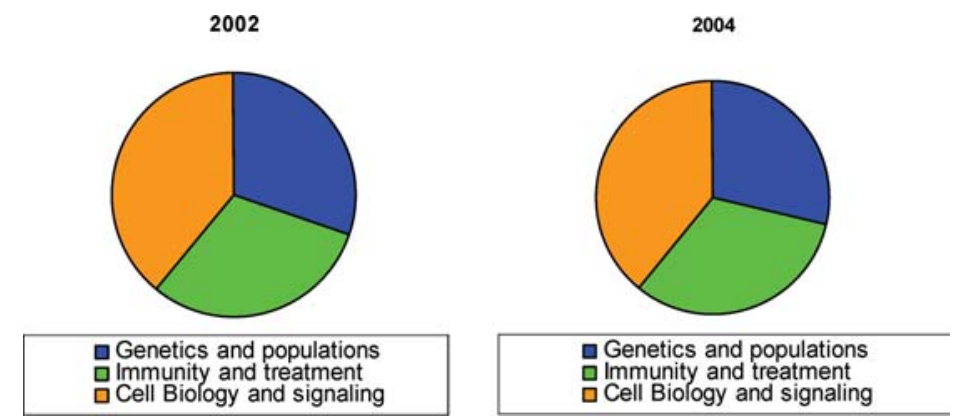

Figure 2. Charts depict distribution of abstracts at 2002 and 2004 conferences based on the division of abstracts selected in 2002.

also a natural target of study. Certainly the acquisition of drug resistance is one of the hallmarks of a biofilm. Several laboratories are studying the transcriptional and proteomic profiling of biofilms. Dr. D'Enfert nicely demonstrated that gene profiles are similar irrespective of the model chosen. This makes life easier at the bench, but what does it mean biologically? The biofilm is a complex structure with many layers including substrate adherence, cell-cell agglutination, differentiation, etc. Therefore, the biofilm comprises multiple cell populations which will have different transcription profiles. Experiments will have to be creatively designed to dissect the different layers of biofilms and which are important to focus on at a clinical level. Drs. Mitchell and Iraqui have used mutational analysis in C. albicans and C. glabrata respectively, to identify specific genes required for biofilm formation. Dr. Mitchell has taken this one step further and developed an elegant approach to determine at which stage specific genes are required. Questions are beginning to be answered in vitro but what about in vivo? Dr. Ghanoum has developed an in vivo catheter model in rabbits, which will aid in answering biologically relevant questions. Do biofilms ever consist of only one species? Additional species will add a new level of complexity. Indeed, Dr. Hogan, showed that Psueudomonas secretes a quorum sensing factor that inhibits filamentation in Candida. This protects Candida from Psuedomonas, but what about the biofilm? Studies with Candida alone suggest that filamentation is required for optimal biofilm formation.

Filamentation: long the love of Candida researchers. Drs. Berman and Sudbery have certainly convinced us that pseudohyphae are a separate developmental stage from hyphae and in fact, are more similar to yeast than hyphae. But what is the biological significance? All stages are found in vivo. Pseudohyphae and hyphae express different proteins; does this affect virulence? Would it help to shift the balance of Candida morphological types? Previous meetings have exposed us to the multiple signaling pathways that Candida uses to filament and focused on individual genes in the different pathways. Although additional components continue to be identified, transcriptome analysis is beginning to distinguish the signaling cascades that are associated with different cellular processes. We know environmental conditions including temperature, $\mathrm{pH}$, serum, $\mathrm{CO}_{2}$ and embedment induce filamentation. We are beginning to identify specific molecules that are/ not involved in pathway sensing. The quorum sensing factor, Farnesol, which inhibits filamentation, appears to act through the histidine kinase pathway. The response to $\mathrm{CO}_{2}$ is not through aquaporin. However, the specific response elements of these pathways remain a mystery. As with drug resistance, laboratories are focusing on what regulates these different pathways; are they co-regulated? (M. Kruppa, F. Mählschlegel, T. Doedt, J. Morschhäuser). Adapting all these separate pathways to cellular processes is now the rage. We can look at transcriptional profiles during biofilm formation, filamentation, phagocytosis, response to stresses, programmed cell death, and response in vitro to in vivo simulated environmental conditions. These allow the assignment of pathways, particularly metabolic and biosynthetic pathways to specific cellular processes. These studies often show that common genes are regulated during stressful conditions. Several laboratories reported studies of 
transcriptional analysis during C. albicans: host cell interactions. In several instances similar global responses were seen. For instance, glyoxylate pathway genes were induced during phagocytosis. Activation of apparent contradictory pathways may indicate the presence of several stages during the incubation period. For instance, Drs. Lorenz and Hube demonstrated that both the glyoxylate and glycolytic cycles were activated when Candida was incubated with macrophages and blood, respectively. Further studies demonstrated that Candida uses the glyoxylate cycle while being phagocytosed and the expression of glycolytic cycle genes corresponded to extracellular stages. These results correspond with those seen under specific filamenation conditions eg upregulation of glycolytic and metabolic pathways. However, in other situations, global responses are not significantly the same. This was demonstrated by Dr. Park who showed that quite different profiles are seen in C. albicans dependent on whether they associate with epithelial or endothelial cells. Another large group of genes expressed under all conditions were the "unknown" genes. Can we learn anything by comparing the expression pattern of unknown genes in the different cellular processes being profiled? This could be particularly interesting, since the percentage of unknown genes appears to be higher when addressing host-cell interactions. Hopefully, these studies will give us a better understanding of what pathways are regulated during pathogenesis.

In 2002, transcriptome analysis of $C$. albicans was in its infancy. In part, this was due to the absence of available microarrays, as well as incomplete sequencing of the genome. Dr. Whiteway announced the availability of the Candida microarray and soon after Qiagen made an oligo array commercially available. Additionally, NIH funded multiple project grants to construct microarrays. Consequently, at this meeting, transcriptome analysis was a constituent of a great number of talks and posters. These global analyses will take a while to completely decipher but the community is well on the way to the next challenge: proteomics. Proteomics has begun to be used in various laboratories to look at global responses. Dr. A. Brown presented the Aberdeen Proteomics Service Facility of COGEME (http://www.abdn.ac.uk/cogeme).
Several laboratories have modified protein extraction conditions to specifically look at cell wall proteins (C. Nombela, P. deGroot). Dr. C. Nombela has posted their 2-D maps on the World Wide Web (http://babbage.scs.ucm.es/2d/ $2 \mathrm{~d} . h \mathrm{tml})$. The cell wall is obviously important, being the first thing the host sees but is often difficult to dissect. The advent of proteomics will hopefully assist in the mystery of cell wall assembly and potential identification of vaccine targets. Proteomics will give a global picture of which proteins are expressed under certain conditions. Additional experimental procedures, e.g., TAP purifications will specifically identify proteins in complexes. The compilation of these studies and correlation with transcriptome analyses will hopefully enlighten us on the working of the C. albicans cell during pathogenesis.

After 16 years of Candida and Candidiasis meetings, we certainly know and understand much more about the functioning of the organism. On the flip side, we also realize how limited that knowledge really is. Most dramatically we are not much improved in our ability to diagnose candidiasis. This is quite evident by the overviews of Candida and candidiasis and clinical presentations. Candida research is still skewed toward gene bashing. However the presentations indicated that the genetic studies are graduating toward more focused and controlled questions to address host:organism interations. This time around there were significantly fewer slides depicting Southern analyses and an disordinate percentage of Venn diagrams and Pie graphs. However, it is just these depictions of global cellular processes instead of specific genes that will better aid in understanding the infectious process and identification of drug targets and therapeutic treatments. Researchers in Candida or candidiasis are challenged to ask questions that will integrate "gene bashing" and "host defense". Consequently, the next conference will truly address studies in Candida and candidiasis.

\section{References}

1. Names of presenters are listed as a group after a summation of the topic area. More information can be obtained from the conference abstract book (details in references). It is realized that not all presenters have been acknowledged and for this the author apologizes. 
2. Jones T, Federspiel N A, Chibana H, Dungan J, Kalman S, Magee B B, Newport G, Thorstenson Y R, Agabian N, Magee $\mathrm{P}$ T, Davis R W, Scherer $\mathrm{S}$. The diploid genome sequence of Candida albicans. Proc Natl Acad sci U $S A$ 2004; 101: 7329-7334.

3. Hull c M, Johnson A D. Identification of mating type-like locus in the asexual pathogenic yeast Candida albicans. Scinece 1999; 285: 1271-1275.

4. Hull C M, Raisner R M, Johnson A D. Evidence for mating of the "asexual" yeast Candida albicans in a mammalian host. Science 2000; 289: 307-310.

5. Magee B B, Magee P T. Induction of mating in Candida albicans by construction of MTla and MTLalpha strains. Science 2000;289: 310-313.
6. Lockhart S R, Pujol C, Daniels K J, Miller M G, Johnson A D, Pfaller M A, Soll D R. In Candida albicans, whiteopaque switchers are homozygous for mating type. Genetics 2003; 162: 737-745.

7. Miller MG, Johnson AD. White-opaque switching in Candida albicans is controlled by mating-type locus homeodomain proteins and allows efficient mating. 2002; 110: 293-302.

8. Lockhart SR, Daniels KJ, Zhao R, Wessels D, Soll DR. Cell biology of mating in Candida albicans. Eukaryot Cell 2003; 2: 49-61.

Address for correspondence: J. Sturtevant, Louisiana State University, LA 70119, New Orleans, USA

E-mail: jsturt@1suhsc.edu 